\title{
Estimation Schemes for Networked Control Systems Using UDP-Like Communication
}

\author{
Michael Epstein, Ling Shi and Richard M. Murray
}

\begin{abstract}
In this work we consider a class of networked control systems (NCS) when the control signal is sent to the plant via a UDP-like communication protocol, the controller sends a communication packet to the plant across a lossy network but the controller does not receive any acknowledgement signal indicating the status of reception/delivery of the control packet. Standard observer based estimators assume the estimator has knowledge of what control signal is applied to the plant, but under the UDP-like communication scheme the estimator does not know what control is applied. Continuing previous work, we present a simple estimation algorithm consisting of a state estimator and mode observer. For single input systems we can add an extra control signal that guarantees recovery of the fate of the control packet. Using a modified state feedback with the added input we can guarantee the estimation error is bounded as is the expected value of the state. This extra input is removed and sufficient conditions on the system properties are given to assure the estimation remain bounded. Comparisons are made between the algorithm presented and the method of unknown input observer. Simulations are provided to demonstrate the algorithm.
\end{abstract}

\section{INTRODUCTION}

In recent years networked control systems (NCS) have gained much attention in the research community. Networked control systems are characterized by the presences of communication links in the feedback loop. These communication links introduce delays and losses of information in the feedback loop that can degrade the performance of the control system. The effect of information loss is considered in this paper.

In [1] Sinopoli et al began looking at closing the loop across lossy networks. They inserted a lossy network between the controller/estimator and the actuators/plant and attempted to solve the LQG problem in this framework. In this work they made the implicit assumption that the estimator/controller had direct knowledge about the fate of the control packet sent to the plant by way of an acknowledgement signal, i.e. a TCP-like protocol. They used this assumption to show that a separation principle holds and that the optimal LQG control is linear with a bounded cost when the percentage of loss is below a threshold.

Sinopoli et al began to consider UDP-like communication protocols, where there is no receive acknowledgement, for the network between the controller/estimator and the acutators/plant in [2], [3]. They show that in this setting the LQG controller is in general nonlinear and cannot, in general, be found in closed form. Other researchers have studied

Control and Dynamical Systems, California Institute of Technology, Pasadena CA 91125 epstein, shiling, murray ecds.caltech.edu the effects of using UDP-like communication protocols in NCS [4], [5]. While sending control commands without an acknowledgement signal will make the analysis more difficult and could require modifications to standard control algorithms, there is evidence that using the UDP protocol is advantageous over the TCP protocol due to lower latency, less overhead in terms of packet length and decreased software complexity [6], [7]. We investigate a particular case of NCS using UDP-like communication to transmit control signals to a remote plant.

We assume there is perfect communication between the sensors and the estimator/controller so that the measurement data is always available at the estimator. The network connecting the estimator/controller to the actuators/plant uses UDP-like protocols. This setup is summarized in Fig. 1. We will present an estimator algorithm guaranteed to recover the fate of the control packet. The estimator algorithm consists of state and mode observers as well as a constraint on the control signal. Removing the constraint on the control signal we will no longer be guaranteed to recover the fate of the packet, but we will show that under certain conditions the estimation error has a finite upper bound. We will show how this algorithm can be used with a modified state feedback controller to stabilize the closed loop system.

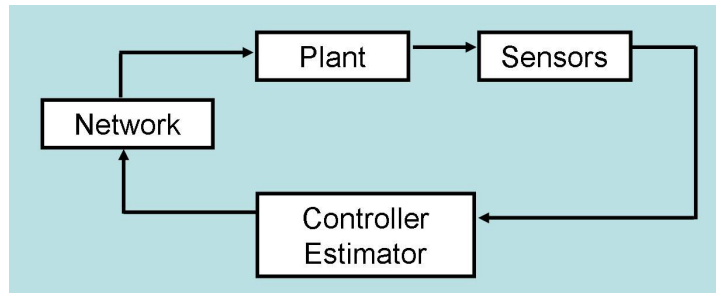

Fig. 1. NCS feedback loop.

The work presented in this paper is an extension of [8]. With the same problem setting and using the same algorithm we expand the previous analysis to show stability and provide a bound on the expected error when system noise is present. We further modify the previous algorithm removing the constraint that the control input need to be large enough to be detected and give stability conditions for this case. These conditions are then shown to be comparable to that for the so called unknown input observer.

The paper is organized as follows. In Section II we set up the problem in a mathematical framework. The proposed estimator algorithm and its convergence properties are presented in Section III as well as the well known unknown 
input observer which is compared to our algorithm. Examples are given in Section IV to illustrate the theory.

\section{Problem Set UP}

We consider a networked control system where the controller sends commands to the actuator across a packet dropping network as in Fig. 1. The network is assumed to be following UDP-type protocol. That is the controller sends signals to the actuators but does not receive any form of acknowledgement, i.e. the controller does not know if the packet was dropped or not.

The plant we consider is a discrete-time linear system. If the control packet is not received it is assumed the plant applies no control and evolves open loop. The plant dynamics

$$
\begin{aligned}
x_{k+1} & =A x_{k}+\gamma_{k} B u_{k}+w_{k} \\
y_{k} & =C x_{k}+v_{k}
\end{aligned}
$$

where $x_{k} \in \mathbb{R}^{n}$ is the state vector, $u_{k} \in \mathbb{R}^{r}$ the control input and $y_{k} \in \mathbb{R}^{m}$ the sensor output. The process noise is given by $w_{k} \in \mathbb{R}^{n}$ and the measurement noise by $v_{k} \in \mathbb{R}^{m}$, which will be assumed to be bounded. The variable $\gamma_{k} \in\{0,1\}$ indicates if the packet containing $u_{k}$ was received at the plant $\left(\gamma_{k}=1\right)$ or if it was dropped $\left(\gamma_{k}=0\right)$. It is assumed if the packet is dropped the plant applies no control for that time step, i.e. it evolves open loop. Note also there is no network between the plant/sensors and the estimator/controller, so measurements are always available. We further assume $A$ is unstable, $(A, B)$ is controllable and $(A, C)$ is observable, so that in the absence of the network $F$ and $L$ are designed to make $A+B F$ and $A-L C A$ stable.

We also make the assumption that $\operatorname{rank}(C B)=$ $\operatorname{rank}(B)=r \leq n$. This will be required as we desire to recover the fate of $\gamma_{k}$ at time $k+1$, meaning we need the effect of $\gamma_{k}$ be present in

$$
y_{k+1}=C x_{k+1}=C A x_{k}+\gamma_{k} C B u_{k}+d_{k},
$$

where $d_{k}=C w_{k}+v_{k+1}$. If the rank condition on $C B$ did not hold then it is possible the $\gamma_{k}$ would disappear from $y_{k+1}$. Physically this condition can be interpreted as requiring any states that are directly affected by the input be measured.

Under the UDP-like communication scheme we are considering, the estimator has no knowledge about the value $\gamma_{k}$. Therefore an observer for this system could take the form of

$$
\hat{x}_{k+1}=A \hat{x}_{k}+\hat{\gamma}_{k} B u_{k}+L\left(y_{k+1}-C A \hat{x}_{k}-\hat{\gamma}_{k} C B u_{k}\right)
$$

where the decision must be made how to select $\hat{\gamma}_{k}$.

Writing the estimation error as $e_{k}=x_{k}-\hat{x}_{k}$ we see it evolves according to

$$
e_{k+1}=(A-L C A) e_{k}+\left(\gamma_{k}-\hat{\gamma}_{k}\right)(B-L C B) u_{k}+z_{k}
$$

where we have used the notation $z_{k}=w_{k}-L d_{k}$. Clearly if $\hat{\gamma}_{k}=\gamma_{k}$ then and (ignoring the noise terms) the estimation error will evolve as $e_{k+1}=(A-L C A) e_{k}$ which is stable. Recalling the UDP-like communication protocol, however, the estimator receives no acknowledgement and hence does not know the value of $\gamma_{k}$ when deciding on $\hat{\gamma}_{k}$. As a result the estimator could either try to reason about $\gamma_{k}$ (if possible) or simply set $\hat{\gamma}_{k}$ to a predetermined value. The case of preselecting a value for $\hat{\gamma}_{k}$ is referred to as the naive schemes and was analyzed in [8].

For any system we design, the state will evolve open loop when $\gamma_{k}=0$, but if we were able to design a system that could recover $\hat{\gamma}_{k}=\gamma_{k}$ at time $k+1$, then the estimation error will be indifferent to the packet drops and we return to the TCP case and can tolerate a higher percentage of drops. The goal is to design an estimator and a control algorithm that can either recover the fate of $\gamma_{k}$ at time $k+1$ or not be affected by the value of $\gamma_{k}$. The options analyzed in this paper consist of the algorithm presented in [8] which tries to recover the value of $\gamma_{k}$ and the unknown input observer [9] which completely removes the control term, and hence $\gamma_{k}$, from $e_{k}$.

\section{Estimation Algorithm}

As stated above it is clear that we seek an estimator scheme that can recover $\hat{\gamma}_{k}=\gamma_{k}$ as this would make the NCS revert to the TCP-like communication protocol. As first presented in [8] we will use an estimator algorithm consisting of the state observer from Eqn. (3) and choosing $\hat{\gamma}_{k}$ according to the mode observer

$$
\hat{\gamma}_{k}=\arg \min _{\beta \in\{0,1\}}\left\|y_{k+1}-C A \hat{x}_{k}-\beta C B u_{k}\right\|^{2} .
$$

The norm $\|\cdot\|$ above, and all other norms in this paper, are assumed to be the 2-norm unless otherwise explicitly stated. We will also use the $H$-norm, defined for some positive definite matrix $H$. For a matrix $X$ we have $\|X\|_{H}=$ $\left\|H^{1 / 2} X H^{-1 / 2}\right\|$ and for a vector $x$ it is $\|x\|_{H}=\sqrt{x^{T} H x}$.

The mode observer above can be shown to recover the true state of $\gamma_{k}$ under the conditions below.

Proposition 1: For the mode observer that chooses $\hat{\gamma}_{k}$ according to Eqn. (5) the following statements hold

- If $u_{k}^{T} B^{T} C^{T} C B u_{k}>2\left|u_{k}^{T} B^{T} C^{T}\left(C A e_{k}+d_{k}\right)\right|$ then $\hat{\gamma}_{k}=\gamma_{k}$

- If $\hat{\gamma}_{k} \neq \gamma_{k}$ then $u_{k}^{T} B^{T} C^{T} C B u_{k}<2\left|u_{k}^{T} B^{T} C^{T}\left(C A e_{k}+d_{k}\right)\right|$

Proof: Returning to Eqn. (5) we can write

$$
\begin{aligned}
\| y_{k+1}= & C A \hat{x}_{k}-\beta C B u_{k} \|^{2} \\
= & \left\|C A x_{k}+\gamma_{k} C B u_{k}+d_{k}-C A \hat{x}_{k}-\beta C B u_{k}\right\|^{2} \\
= & \left\|C A e_{k}+d_{k}+\left(\gamma_{k}-\beta\right) C B u_{k}\right\|^{2} \\
= & e_{k}^{T} A^{T} C^{T} C A e_{k}+d_{k}^{T} d_{k} \\
& +\left(\gamma_{k}-\beta\right)^{2} u_{k}^{T} B^{T} C^{T} C B u_{k} \\
& +2\left(\gamma_{k}-\beta\right) u_{k}^{T} B^{T} C^{T}\left(C A e_{k}+d_{k}\right)
\end{aligned}
$$

Since $e_{k}^{T} A^{T} C^{T} C A e_{k}+d_{k}^{T} d_{k} \geq 0$ and is independent of $\left(\gamma_{k}, \beta\right)$ we can remove it from the minimization, that leaves

$$
\begin{aligned}
& J\left(\gamma_{k}, \beta\right)=\left(\gamma_{k}-\beta\right)^{2} u_{k}^{T} B^{T} C^{T} C B u_{k} \\
&+ 2\left(\gamma_{k}-\beta\right) u_{k}^{T} B^{T} C^{T}\left(C A e_{k}+d_{k}\right) .
\end{aligned}
$$


Recall that $\gamma_{k} \in\{0,1\}$ and $\beta \in\{0,1\}$, so if $\beta=\gamma_{k}$ then $J=0$ otherwise $J=u_{k}^{T} B^{T} C^{T} C B u_{k}+2\left(\gamma_{k}-\right.$ B) $u_{k}^{T} B^{T} C^{T}\left(C A e_{k}+d_{k}\right)$. Clearly since $J$ is being minimized, a sufficient condition to choose $\gamma_{k}$ correctly is

$$
u_{k}^{T} B^{T} C^{T} C B u_{k} \pm 2 u_{k}^{T} B^{T} C^{T}\left(C A e_{k}+d_{k}\right)>0
$$

and since both terms are scalars this is equivalent to the first condition. The second condition is a result that if $\hat{\gamma}_{k} \neq \gamma_{k}$ then $J<0$ which is only possible if the inequality holds.

For the remainder of the paper we will focus our attention on single input systems, where $u_{k} \in \mathbb{R}$ is a scalar. This means also that $B^{T} C^{T} C B \in \mathbb{R}$ is a scalar quantity and we have $u_{k}^{T} B^{T} C^{T} C B u_{k}=u_{k}^{2} B^{T} C^{T} C B$. Let

$$
\Lambda=\frac{1}{B^{T} C^{T} C B} B^{T} C^{T}
$$

which allows us to restate Proposition 1 as

- $\left|u_{k}\right|>2\left|\Lambda\left(C A e_{k}+d_{k}\right)\right| \Rightarrow \hat{\gamma}_{k}=\gamma_{k}$

- $\hat{\gamma}_{k} \neq \gamma_{k} \Rightarrow\left|u_{k}\right|<2\left|\Lambda\left(C A e_{k}+d_{k}\right)\right|$

From Eqn. (8) we have a sufficient condition on $\left(u_{k}, e_{k}, d_{k}\right)$ to assure $\hat{\gamma}_{k}=\gamma_{k}$. If we knew $e_{k}$ and $d_{k}$, we could simply pick $u_{k}$ to satisfy the condition but of course these are unknown. Instead assume we do know a bound on the initial conditions and that we consider only norm bounded noises

$$
\left\|x_{0}\right\| \leq \delta_{x},\left\|e_{0}\right\| \leq \delta_{e},\left\|d_{k}\right\| \leq \delta_{d},\left\|z_{k}\right\| \leq \delta_{z} .
$$

The estimation error from the state observer given in Eqn. (4) can be evaluated at time $k$ according to

$$
e_{k}=(A-L C A)^{k} e_{0}+\sum_{j=0}^{k-1}(A-L C A)^{k-j-1} h_{j}
$$

with $h_{j}=\left(\gamma_{j}-\hat{\gamma}_{j}\right)(B-L C B) u_{j}+z_{j}$. If we assume that we are always able to pick a $u_{k}$ satisfying $\left|u_{k}\right|>$ $2\left|\Lambda\left(C A e_{k}+d_{k}\right)\right|$ then $\hat{\gamma}_{k}=\gamma_{k}$ and the norm of the estimation error can be bounded according to

$$
\left\|e_{k}\right\| \leq \eta_{k} \triangleq\left\|(A-L C A)^{k}\right\| \delta_{e}+\sum_{j=0}^{k-1}\left\|(A-L C A)^{k-j-1}\right\| \delta_{z} .
$$

\section{A. Augmenting the Control Signal to Guarantee Detection}

In this section we show that by using a modified state feedback with an augmented control signal we can not only guarantee detection of $\gamma_{k}$, i.e. $\hat{\gamma}_{k}=\gamma_{k}$, but also that the closed loop will be stable in some sense.

Proposition 2 (From [8]): If we pick at each time step a control value that satisfies

$$
\left|u_{k}\right|>\Delta_{k} \triangleq 2\|\Lambda\|\left(\|C A\| \eta_{k}+\delta_{d}\right)
$$

then we are guaranteed to have $\hat{\gamma}_{k}=\gamma_{k}$. In addition the right hand side of Eqn. (10) will remain upperbounded by a finite value as $k \rightarrow \infty$.
Proof: See [8].

We now have a state and mode observer together with a constraint on the control action to ensure the fate of the $k^{\text {th }}$ control packet, $\gamma_{k}$, can be recovered at time $k+1$. This assures the estimation error will be bounded. The algorithm is summarized in Fig. 2.

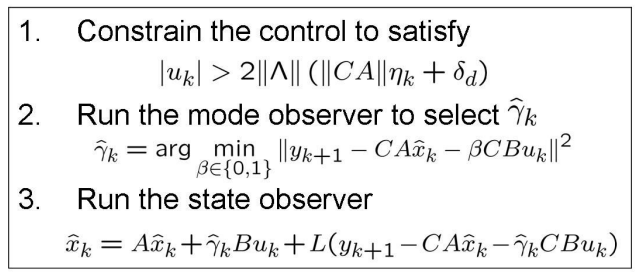

Fig. 2. Estimator Algorithm.

We are also interested in the closed loop performance of the system. In [8] we showed that in the noise-free case we can achieve almost sure stability using the estimator described above along with a slightly modified state feedback controller of the form

$$
u_{k}=F \hat{x}_{k}+\operatorname{sgn}\left(F \hat{x}_{k}\right) 2\|\Lambda\|\|C A\|\left\|(A-L C A)^{k}\right\| \delta_{e} .
$$

It is straightforward to show that in the case with no noise, i.e. $w_{k}=v_{k}=\delta_{z}=\delta_{d}=0$, that Eqn. (11) will satisfy the condition in Eqn. (10) meaning the estimator algorithm is guaranteed to provide $\hat{\gamma}_{k}=\gamma_{k}$. In [8] it is shown that if $A+B F$ is stable, the closed loop system is almost surely stable if for $\bar{\gamma}=\mathbf{E}\left[\gamma_{k}\right]$ there exists some $H$-norm such that

$$
\left\|\left[\begin{array}{c|c}
A_{B F} & -B F \\
\hline 0 & A_{L C}
\end{array}\right]\right\|_{H}^{\bar{\gamma}} \cdot\left\|\left[\begin{array}{c|c}
A & 0 \\
\hline 0 & A_{L C}
\end{array}\right]\right\|_{H}^{1-\bar{\gamma}}<1,
$$

with $A_{B F}=A+B F$ and $A_{L C}=A-L C A$. Below we extend the results of [8] to the case where the bounded system and measurement noise is present, i.e. $\delta_{z}, \delta_{d}>0$.

Theorem 3: Using the estimator described in Fig. 2 along with a modified state feedback controller

$$
u_{k}=F \hat{x}_{k}+\operatorname{sgn}\left(F \hat{x}_{k}\right) \Delta_{k}
$$

then if $\gamma_{k}$ is an i.i.d random variable with $\mathbf{E}\left[\gamma_{k}\right]=\bar{\gamma}$, and there exists a positive definite matrix $H>0$ such that

$$
\psi \triangleq(1-\bar{\gamma}) \cdot\|A\|_{H}+\bar{\gamma} \cdot\|A+B F\|_{H}<1
$$

then the following holds

$$
\mathbf{E}\left[\left\|x_{k}\right\|\right] \leq\left(\psi^{k} \delta_{x}+\frac{1-\psi^{k}}{1-\psi} \cdot \Sigma\right) \frac{\bar{\lambda}_{H}}{\underline{\lambda}_{H}}
$$

with

$$
\Sigma \triangleq \delta_{w}+\max _{k \geq 0}\|B\| \Delta_{k}+\|B F\| \eta_{k},
$$

and $\bar{\lambda}_{H}$ and $\underline{\lambda}_{H}$ signifying the maximum and minimum eigenvalues of $H$ respectively. 
Proof: Using the control signal in Eqn. (12) the state dynamics update equation can then be rewritten as

$$
\begin{aligned}
x_{k+1} & =\left(A+\gamma_{k} B F\right) x_{k}+q_{k} \\
q_{k} & =w_{k}+\gamma_{k} B\left(\operatorname{sgn}\left(F \hat{x}_{k}\right) \Delta_{k}-F e_{k}\right) .
\end{aligned}
$$

Using the control signal in Eqn. (12) with the estimator algorithm guarantees that $\hat{\gamma}_{k}=\gamma_{k}$ and thus the error is bounded according to Eqn. (9) and we see

$$
\left\|q_{k}\right\| \leq \delta_{w}+\|B\| \Delta_{k}+\|B F\| \eta_{k} \leq \Sigma
$$

Now define $\mathcal{A}_{j}^{k}=\prod_{i=j}^{k-1}\left(A+\gamma_{i} B F\right)$ with $\mathcal{A}_{j}^{k}=I$ if $j \leq k$, this allows us to express the state as

$$
x_{k}=\mathcal{A}_{0}^{k} x_{0}+\sum_{j=0}^{k} \mathcal{A}_{j+1}^{k} q_{j} .
$$

Taking the expectation of the $H$-norm of this expression above we get

$$
\begin{aligned}
\mathbf{E}\left[\left\|x_{k}\right\|_{H}\right] & =\mathbf{E}\left[\left\|\mathcal{A}_{0}^{k} x_{0}+\sum_{j=0}^{k} \mathcal{A}_{j+1}^{k} q_{j}\right\|_{H}\right] \\
& \leq \mathbf{E}\left[\left\|\mathcal{A}_{0}^{k} x_{0}\right\|_{H}\right]+\sum_{j=0}^{k} \mathbf{E}\left[\left\|\mathcal{A}_{j+1}^{k} q_{j}\right\|_{H}\right](17)
\end{aligned}
$$

Examining the first term we can write

$$
\begin{aligned}
\mathbf{E}\left[\left\|\mathcal{A}_{0}^{k} x_{0}\right\|_{H}\right] & \leq \mathbf{E}\left[\left\|\mathcal{A}_{0}^{k}\right\|_{H} \cdot\left\|x_{0}\right\|_{H}\right] \\
& \leq \mathbf{E}\left[\left\|\mathcal{A}_{0}^{k}\right\|_{H}\right] \cdot \delta_{x} \cdot \bar{\lambda}_{H} \\
& =\mathbf{E}\left[\left\|\prod_{j=0}^{k}\left(A+\gamma_{j} B F\right)\right\|_{H}\right] \delta_{x} \cdot \bar{\lambda}_{H} \\
& \leq \mathbf{E}\left[\prod_{j=0}^{k}\left\|\left(A+\gamma_{j} B F\right)\right\|_{H}\right] \delta_{x} \cdot \bar{\lambda}_{H} \\
& \leq \prod_{j=0}^{k} \mathbf{E}\left[\left\|\left(A+\gamma_{j} B F\right)\right\|_{H}\right] \delta_{x} \cdot \bar{\lambda}_{H}(18)
\end{aligned}
$$

where the last line comes from the assumption that the packet drops are independent from one time step to the next. Note that the following holds

$\mathbf{E}\left[\left\|A+\gamma_{j} B F\right\|_{H}\right]=(1-\bar{\gamma}) \cdot\|A\|_{H}+\bar{\gamma} \cdot\|A+B F\|_{H}=\psi$

thus we have

$$
\mathbf{E}\left[\left\|\mathcal{A}_{0}^{k} x_{0}\right\|\right] \leq \psi^{k} \delta_{x} \cdot \bar{\lambda}_{H} .
$$

Now returning to the second term in Eqn. (17)

$$
\begin{array}{rl}
\sum_{j=0}^{k} & \mathbf{E}\left[\left\|\mathcal{A}_{j+1}^{k} q_{j}\right\|_{H}\right] \\
& \leq \sum_{j=0}^{k} \mathbf{E}\left[\left\|\mathcal{A}_{j+1}^{k}\right\|_{H} \cdot\left\|q_{j}\right\|_{H}\right] \\
& \leq \sum_{j=0}^{k} \prod_{i=j+1}^{k} \mathbf{E}\left[\left\|\left(A+\gamma_{i} B F\right)\right\|_{H}\right] \cdot \Sigma \cdot \bar{\lambda}_{H} \\
& \leq \sum_{j=0}^{k} \prod_{i=j+1}^{k} \psi \cdot \Sigma \cdot \bar{\lambda}_{H} \\
& \leq \sum_{j=0}^{k} \psi^{k-j} \cdot \Sigma \cdot \bar{\lambda}_{H} \\
& =\frac{1-\psi^{k}}{1-\psi} \cdot \Sigma \cdot \bar{\lambda}_{H} .
\end{array}
$$

Then noting $\left\|x_{k}\right\| \leq\left\|x_{k}\right\|_{H} / \underline{\lambda}_{H}$ and combining this with Eqns. (19) and (20) in Eqn. (17) we arrive at the expression in Eqn. (14).

To evaluate the performance as $k \rightarrow \infty$ it is easy to see that since $\psi<1$ we have

$$
\lim _{k \rightarrow \infty} \mathbf{E}\left[\left\|x_{k}\right\|\right]=\frac{\Sigma}{1-\psi} \cdot \frac{\bar{\lambda}_{H}}{\underline{\lambda}_{H}} .
$$

Remark 4: The stability shown in the theorem above differs from other current stability results [10] as those require the noise signal to be an $l_{2}$ sequence, while clearly the noise in Eqn. (16) is not.

\section{B. Removing the Added Input Signal}

As shown above by combining the estimator algorithm with the modified state feedback control signal in Eqn. (12) the state is stable in expectation. The question arises whether it is necessary to include the extra control effort term $\Delta_{k}$. Without this extra term it is no longer possible to guarantee that $\hat{\gamma}_{k}=\gamma_{k}$ but as we see below it might still be possible to stabilize the plant. We begin by showing that the estimation error will be bounded even if the fate of $\gamma_{k}$ is not always recovered.

Lemma 5: If there exists an observation gain $L$ and a positive definite matrix $P>0$ such that for all $\alpha_{k} \in[0,1]$

$$
\overline{\Delta V}_{k}=\left(\tilde{A}_{k}-L \tilde{C}_{k}\right)^{T} P\left(\tilde{A}_{k}-L \tilde{C}_{k}\right)-P<0
$$

with

$$
\begin{aligned}
& \tilde{A}_{k}=A-\alpha_{k} 2 B \Lambda C A \\
& \tilde{C}_{k}=C A-\alpha_{k} 2 C B \Lambda C A
\end{aligned}
$$

then using any control signal the estimation error will be upper bounded according to

$$
\left\|e_{k}\right\| \leq E \triangleq \sqrt{\frac{\lambda_{2}}{\lambda_{1}}}\left(1+\lambda_{6} \frac{\lambda_{5}+\sqrt{\lambda_{5}^{2}+\lambda_{2} \lambda_{3}}}{\lambda_{3}}\right) R
$$


with

$$
\begin{aligned}
\lambda_{1} & =\underline{\lambda}(P) \\
\lambda_{2} & =\bar{\lambda}(P) \\
\lambda_{3} & =\min _{0 \leq \alpha_{k} \leq 1} \underline{\lambda}\left(-\overline{\Delta V}_{k}\right) \\
\lambda_{4} & =\max _{0 \leq \alpha_{k} \leq 1} \bar{\lambda}\left(-\overline{\Delta V}_{k}\right) \\
\lambda_{5} & =\max _{0 \leq \alpha_{k} \leq 1}\left\|\left(\tilde{A}_{k}-L \tilde{C}_{k}\right)^{T} P\right\| \\
\lambda_{6} & =\max _{0 \leq \alpha_{k} \leq 1}\left\|\tilde{A}_{k}-L \tilde{C}_{k}\right\| \\
R & =2\|(B-L C B) \Lambda\| \delta_{d}+\delta_{z}
\end{aligned}
$$

where $\bar{\lambda}(X)$ and $\underline{\lambda}(X)$ signify the largest and smallest eigenvalue of some matrix $X$ respectively.

Proof: Define $\Omega_{k}=\left(\gamma_{k}-\hat{\gamma}_{k}\right)(B-L C B) u_{k}$ and consider the possible combinations of $\left(\gamma_{k}, \hat{\gamma}_{k}\right)$. If $\gamma_{k}=\hat{\gamma}_{k} \Rightarrow \Omega_{k}=0$. From Proposition 1 if $\gamma_{k} \neq \hat{\gamma}_{k}$ the following must hold

$$
u_{k}^{T} B^{T} C^{T} C B u_{k}<-2\left(\gamma_{k}-\hat{\gamma}_{k}\right) u_{k}^{T} B^{T} C^{T}\left(C A e_{k}+d_{k}\right) .
$$

Let us first consider the case where $\left(\gamma_{k}, \hat{\gamma}_{k}\right)=(0,1)$. Since $u_{k}$ is a scalar Eqn. (32) reduces to $u_{k}^{2}<u_{k} \cdot 2 \Lambda \cdot\left(C A e_{k}+d_{k}\right)$ requiring $u_{k}$ and $\Lambda \cdot\left(C A e_{k}+d_{k}\right)$ to have the same sign. If $u_{k}>0 \Rightarrow 0<u_{k}<2 \Lambda \cdot\left(C A e_{k}+d_{k}\right)$ and if $u_{k}<$ $0 \Rightarrow 2 \Lambda \cdot\left(C A e_{k}+d_{k}\right)<u_{k}<0$. Combining these two statements we can write $u_{k}=\alpha_{k} 2 \Lambda \cdot\left(C A e_{k}+d_{k}\right)$ for some $\alpha_{k} \in[0,1]$. Similarly it can be shown that for $\left(\gamma_{k}, \hat{\gamma}_{k}\right)=$ $(1,0)$ we have $u_{k}=-\alpha_{k} 2 \Lambda \cdot\left(C A e_{k}+d_{k}\right)$. Incorporating all the possible combinations for $\left(\gamma_{k}, \hat{\gamma}_{k}\right)$ allows us to write $\Omega_{k}=-\alpha_{k}(B-L C B) 2 \Lambda \cdot\left(C A e_{k}+d_{k}\right)$. From Eqn. (4) we can write the error update equation as

$$
\begin{aligned}
e_{k+1} & =(A-L C A) e_{k}+\Omega_{k}+z_{k} \\
& =\left(\tilde{A}_{k}-L \tilde{C}_{k}\right) e_{k}+\alpha_{k}(B-L C B) 2 \Lambda d_{k}+z_{k}
\end{aligned}
$$

which is an uncertain system, through $\left(\tilde{A}_{k}, \tilde{C}_{k}\right)$, with bounded noise, $r_{k}=\alpha_{k}(B-L C B) \Lambda d_{k}+z_{k}$.

Now construct a nonnegative function $V_{k}=e_{k}^{T} P e_{k}$ and using the error dynamics above we see that

$$
\begin{aligned}
\Delta V_{k}= & V_{k+1}-V_{k} \\
= & e_{k+1}^{T} P e_{k+1}-e_{k} P e_{k} \\
= & \left(\left(\tilde{A}_{k}-L \tilde{C}_{k}\right) e_{k}+r_{k}\right)^{T} P\left(\left(\tilde{A}_{k}-L \tilde{C}_{k}\right) e_{k}+r_{k}\right) \\
& -e_{k}^{T} P e_{k} \\
= & e_{k}^{T}\left[\left(\tilde{A}_{k}-L \tilde{C}_{k}\right)^{T} P\left(\tilde{A}_{k}-L \tilde{C}_{k}\right)-P\right] e_{k} \\
& +r_{k}^{T} P r_{k}+2 e_{k}^{T}\left(\tilde{A}_{k}-L \tilde{C}_{k}\right)^{T} P r_{k}
\end{aligned}
$$

the first term is simply $e_{k}^{T} \overline{\Delta V}_{k} e_{k}$ which is negative per Eqn. (21), the term $r_{k}^{T} P r_{k}$ will always be positive and sign of the last term will depend on the vectors $e_{k}$ and $r_{k}$. We would be interested in using $V_{k}$ as a Lyapunov function we need it to decrease along system trajectories, i.e. $\Delta V_{k}<0$, however from Eqn. (34) we see that it is not always guaranteed to be negative. Analyzing the terms in Eqn. (34) further we note

$$
\begin{aligned}
e_{k}^{T} \overline{\Delta V}_{k} e_{k} & \leq-\lambda_{3}\left\|e_{k}\right\|^{2} \\
r_{k}^{T} P r_{k} & \leq \lambda_{2}\left\|r_{k}\right\|^{2} \leq \lambda_{2} R^{2} .
\end{aligned}
$$

Using the Cauchy-Schwarz inequality we see

$$
\begin{aligned}
& \left|e_{k}^{T}\left(\tilde{A}_{k}-L \tilde{C}_{k}\right)^{T} P r_{k}\right| \\
& \quad \leq\left\|e_{k}^{T}\left(\tilde{A}_{k}-L \tilde{C}_{k}\right)^{T} P\right\| \cdot\left\|r_{k}\right\| \leq\left\|e_{k}\right\| \cdot \lambda_{5} R .
\end{aligned}
$$

Combining Eqn. (34) with Eqn.s (35) , (36) , (37) we get

$$
\Delta V_{k} \leq-\lambda_{3}\left\|e_{k}\right\|^{2}+\lambda_{2} R^{2}+2 \lambda_{5} R\left\|e_{k}\right\| .
$$

Note $\Delta V_{k}$ is negative quadratic in $\left\|e_{k}\right\|$, so solving for the positive root we get

$$
e^{*}=\frac{R}{\lambda_{3}}\left(\lambda_{5}+\sqrt{\lambda_{5}^{2}+\lambda_{2} \lambda_{3}}\right)
$$

As $\left\|e_{k}\right\|>e^{*}$ implies $\Delta V_{k}<0$, it is then true that $V_{k+1}<$ $V_{k}$ and $\left\|e_{k+1}\right\|^{2} \leq \frac{V_{k+1}}{\lambda_{1}} \leq \frac{V_{k}}{\lambda_{1}} \leq\left\|e_{k}\right\|^{2} \frac{\lambda_{2}}{\lambda_{1}}$. If $\left\|e_{k}\right\| \leq e^{*}$ it is possible $\Delta V_{k} \geq 0$. We do know, however, that

$$
\left\|e_{k+1}\right\| \leq \lambda_{6}\left\|e_{k}\right\|+R
$$

therefore $\left\|e_{k}\right\| \leq e^{*}$ implies $\left\|e_{k+1}\right\| \leq \lambda_{6} e^{*}+R$. If the actual value of $e_{k+1}$ is such that $\left\|e_{k+1}\right\|>e^{*}$, then $\Delta V_{k+1}<0$ and $\left\|e_{k+2}\right\| \leq\left\|e_{k+1}\right\| \sqrt{\frac{\lambda_{2}}{\lambda_{1}}}$, if the value is $\left\|e_{k+1}\right\| \leq e^{*}$, then $\left\|e_{k+2}\right\| \leq \lambda_{6} e^{*}+R$. Then assuming the initial error also satisfies $\left\|e_{0}\right\| \leq E$ we get the expression in Eqn. (24).

Theorem 6: If there exists an observation gain $L$ and a positive definite matrix $P>0$ satisfying the conditions of Lemma 5. then using a simple state feedback controller without the added effort, i.e. $u_{k}=F \hat{x}_{k}$, the state is bounded in the expected sense

$$
\mathbf{E}\left[\left\|x_{k}\right\|\right] \leq \psi^{k} \delta_{x}+\frac{1-\psi^{k}}{1-\psi} \cdot \Theta
$$

where

$\Theta=\|B F\| \sqrt{\frac{\lambda_{2}}{\lambda_{1}}}\left(\frac{\lambda_{6}}{\lambda_{3}}\right)\left(\lambda_{5}+\sqrt{\lambda_{5}^{2}+\lambda_{2} \lambda_{3}}\right)(1+R)+\delta_{w}$.

Proof: The proof is the same as Theorem 3 without the addition of the $B \Delta_{k}$ term to the bounded noise and the estimation error bounded according to Lemma 5.

Remark 7: The stability conditions presented above are conservative. Performing the worst case analysis for the noise sequences and using the properties of the norm makes the upper bounds conservatives. Furthermore, the conditions are only sufficient not necessary and it turns out the algorithm performs favorably even when the conditions are not satisfied. The value of the derivations above is to show that the algorithms can stabilize the system, as we will show in the simulations below the actual performance can be substantially better than these upper bounds. 


\section{Unknown Input Observers}

The purpose of the estimator algorithm described above is to develop an estimation and control scheme that can tolerate not having information on the fate of the control packets, i.e. $\gamma_{k}$. We now present an alternative estimator that will do the same, namely the well known unknown input observer [9]. It consists of using estimator of the form

$$
\hat{x}_{k+1}=(M A-K C) \hat{x}_{k}+K y_{k}+G y_{k+1}
$$

with $M=I-G C$ and the gains $(K, G)$ must be chosen so that

$$
\begin{aligned}
& \text { - } M B=0 \\
& \text { - } M A-K C \text { is stable }
\end{aligned}
$$

Let $G=B(C B)^{+}+Y\left(I-(C B)(C B)^{+}\right)$satisfy $M B=$ 0 for any arbitrary matrix $Y$ where $(C B)^{+}$is the left (pseudo)inverse of $(C B)$. Applying the unknown input observer to the UDP system the estimation error dynamics using the unknown input observer are given by

$$
e_{k+1}=(M A-K C) e_{k}+w_{k}+K v_{k}+G\left(C w_{k}+v_{k+1}\right) \text {. }
$$

According to [9] the necessary and sufficient conditions for the unknown input observer to exist are that; $\operatorname{rank}(C B)=$ $\operatorname{rank}(B)=r \leq n$, so that $(C B)^{+}$exists, and $(M A, C)$ be detectable.

Lemma 8: The following are true:

1) The existence of a unknown input observer with $M A$ stable implies the conditions for Lemma 5 are satisfied with $L=G$.

2) The existence of an $L$ satisfying the conditions for Lemma 5 implies the existence of a unknown input observer.

Proof:

1) The unknown input observer exists with $M A$ stable and let $L=G$ be the observer gain used in the estimator algorithm. Then since $M B=B-L C B=0$ this means $\left(\tilde{A}_{k}, \tilde{C}_{k}\right)=(A, C)$ and since $M A=$ $A-L C A=\tilde{A}_{k}-L \tilde{C}_{k} \tilde{A}_{k}$ is stable clearly Eqn. (21) can be satisfied.

2) If Eqn. (21) is satisfied for all $\alpha_{k} \in[0,1]$ then it is satisfied for $\alpha_{k}=\frac{1}{2}$ which implies the pair

$$
(A-B \Lambda C A, C A-C B \Lambda C A)
$$

must be detectable. If we let $K=0$ then for the unknown input observer to exist $M A$ must be stable. Since $\operatorname{rank}(C B)=\operatorname{rank}(B)$ we have $(C B)^{+}=$ $\left(B^{T} C^{T} C B\right)^{-1} B^{T} C^{T}$, but as we are considering single input systems $\left(B^{T} C^{T} C B\right)^{-1}$ is a scalar. Thus we can write $M A=A-B \Lambda C A-Y(C A-C B \Lambda C A)$ but since the pair above is detectable $M A$ can be made stable through proper selection of $Y$. Hence the unknown input observer exists.

Thus we see that if the sufficient conditions for the estimator algorithm are satisfied then the unknown input observer exists as well, so the question arises which is better to use? An upper bound for the unknown input observer estimation error can be computed and compared with the upper bound using the estimator algorithm both with and without the added control input. When the necessary and sufficient conditions for the unknown input observer are not satisfied, then by definition constructing an observer that cancels out the unknown input will be unstable, whereas the conditions presented above for upper bound on the estimator algorithm are only sufficient conditions. Thus if the unknown input observer does not exist, although there is no proof that the estimator above will be upper bounded there is also no proof saying it is unstable and it is a better alternative. The example below helps to illustrate the usage of the different schemes.

\section{Simulation ExAmple}

Consider a system in Eqn. (1) - (2) whose dynamics are given by

$$
A=\left[\begin{array}{ll}
1.5 & 0.1 \\
0.3 & 1.3
\end{array}\right], B=\left[\begin{array}{l}
0 \\
1
\end{array}\right], C=\left[\begin{array}{ll}
0 & 1
\end{array}\right]
$$

with $\delta_{x}=\sqrt{2}, \delta_{e}=\sqrt{2}, \delta_{w}=1$ and $\delta_{v}=0.1$. This system has unstable eigenvalues of $[1.2,1.6],(A, B)$ and $(A, C A)$ are controllable and observable, respectively, and $C B=1$ so the estimation algorithm can be used to choose $\hat{\gamma}_{k}$. The estimator algorithm was run with $L=[3.9,0.98]^{T}$ and a state feedback controller with gain $F=[-12.95,-2.05]$.

For this system the unknown input observer does not exist since the pair $(M A, C)$ is not detectable so this method was left out of the simulations. We simulated the UDP estimation algorithm described in this paper with and without the extra control input as well as the naive case of selecting $\hat{\gamma}_{k}=1$ and the TCP case where the estimator has direct knowledge of $\gamma_{k}$. A total of 10,000 simulations were run each for 50 time steps. Random initial conditions and noise sequences were chosen but were the same for all the different estimation schemes. We used an average packet acceptance rate of $\bar{\gamma}=0.85$.

The average of the state and estimation error norms across all 10,0000 simulation is shown in Figure. 3. The scheme using $\hat{\gamma}_{k}=1$ quickly diverges, while as expected the TCP case has the best results. Both UDP estimation schemes, with and without the added input value, show average estimation errors that overlap with the TCP case. In fact when including the additional input value the estimates are identical to the TCP case since we recover $\hat{\gamma}_{k}=\gamma_{k}$, the price to pay is that the state norm is larger as a result of including the extra input term. Using the UDP estimation scheme without the additional input we see that the performance is virtually identical to that of the TCP case for both the estimation error and the state norm. We do not always recover the fate of $\gamma_{k}$ using this scheme, but in fact the selection of $\hat{\gamma}_{k}$ is correct nearly $99 \%$ of the time.

Figure 4 shows the results from a particular simulation. Note how the UDP estimation scheme without the added control tracks the TCP case except for a few time steps starting at 19 when it results in $\hat{\gamma}_{k} \neq \gamma_{k}$. This mistake causes the 

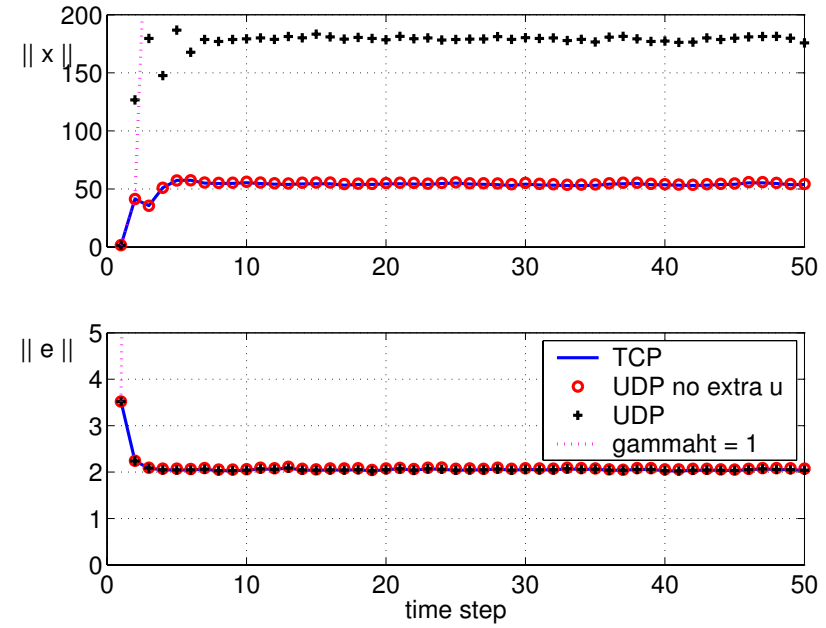

Fig. 3. Average of $\left\|x_{k}\right\|$ and $\left\|e_{k}\right\|$ across all 10,0000 simulations for each of the various estimation schemes.

estimation error to increase slightly which likewise induces a larger state norm, after a few successive time steps with no error in $\hat{\gamma}_{k}$ the estimation error and state norm quickly collapse onto the TCP case. For all the estimation schemes the periods of time where the control packets are dropped, i.e. $\gamma_{k}=0$, correspond to the state norm increasing. The bottom plot shows the control efforts, note how the UDP estimation scheme applies larger control effort because of the larger state norm and the added input used to detect $\gamma_{k}$.

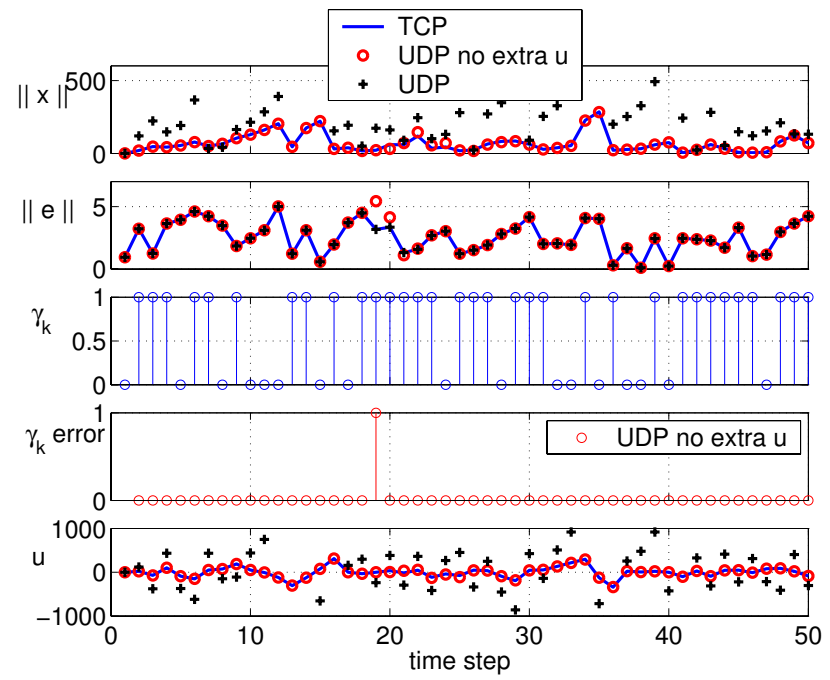

Fig. 4. Plots from a single simulation.

\section{CONClusions And Future Work}

We further analyzed the estimator algorithm first presented in [8] for UDP-like networked control systems. To ensure detection of the fate of the control packet an added control input is included. An upper bound for the expected value of the state norm in the presence of bounded state and measurement noise was presented. If the added control input is removed, the estimator algorithm is no longer guaranteed to detect the fate of the control packet, nonetheless under certain conditions on the system parameters it can still be shown to produce an upper bound to the estimation error which in turn allows an upper bound to the expected of the state norm to be derived. The estimator algorithm is then compared to the unknown input observer which can directly remove the dependence of the control signal on the estimation error. A simulation example shows how the estimator algorithm works well, especially compared to the other methods, even if the sufficient conditions for stability are not satisfied and provides guidelines for designing the estimator in this case.

Since the conditions for stability of the estimator algorithm overlap with those of the unknown input observer it would be good if necessary stability conditions for the estimator algorithm could be derived. Since the algorithm appears to work well even if the sufficient conditions are not satisfied relaxing this conservatism would make the result stronger. Modifying the model to instead include different noise types or uncertainties, having non i.i.d. packet drops and adding intelligence at the plant to apply some predicted control rather than evolve open loop when the control packet is dropped are all areas that can be investigated. Though the results presented here should only need to be modified slightly to include these scenarios. The most interesting extension might be to insert a network between the sensors and estimator, so that the estimator does not always have access to the sensor data, which would most likely require some additional logic in the algorithm.

\section{REFERENCES}

[1] B. Sinopoli, L. Schenato, M. Franceschetti, K. P. M. Jordan, and S. Sastry, "Optimal control with unreliable communication: the tcp case," American Control Conference, 2005.

[2] B. Sinopoli, L. Schenato, M. Franceschetti, K. Poolla, and S. Sastry, "LQG control with missing observation and control packets," 16th IFAC World Congress, 2005.

[3] — , "An LQG optimal linear controller for control systems with packet losses," Conference on Decision and Control, 2005.

[4] C. Robinson and P. Kumar, "Control over networks of unreliable links - controller location and performance bounds," in Proceedings of Control Over Communication Channels (ConComm), 2007.

[5] B. Azimi-Sadjadi, "Stability of networked control systems in the presence of packet losses," in IEEE Conference on Decision and Control, 2003.

[6] N. Ploplys and A. Alleyne, "Udp network communications for distributed wireless control," in American Control Conference, 2003.

[7] M. Das, R. Ghosh, B. Goswami, A. Gupta, A. Tiwari, R. Balasubrmanian, and A. Chandra, "Network control system applied to a large pressurized heavy water reactor," IEEE Trans. on Nuclear Science, vol. 53, pp. 2948-2956, October 2006.

[8] M. Epstein, L. Shi, and R. M. Murray, "An estimation algorithm for a class of networked control systems using udp-like communication schemes," in IEEE Conf. on Decision and Control, 2006.

[9] G. Millerioux and J. Daafouz, "Unknown input observerds for switched linear discrete time systems," in American Control Conference, 2004.

[10] P. Seiler and R. Sengupta, "A bounded real lemma for jump systems," IEEE Trans. Automatic Control, vol. 48, no. 9, 2003. 